\title{
Detailed Balance and Intermediate Statistics
}

\author{
R. Acharya ${ }^{a}$ and P. Narayana Swamy ${ }^{b}$ \\ ${ }^{a}$ Professor Emeritus Physics, Arizona State University, Tempe AZ 85287 \\ ${ }^{b}$ Professor Emeritus Physics, Southern Illinois University, Edwardsville IL 62026
}

\begin{abstract}
We present a theory of particles, obeying intermediate statistics ("anyons"), interpolating between Bosons and Fermions, based on the principle of Detailed Balance. It is demonstrated that the scattering probabilities of identical particles can be expressed in terms of the basic numbers, which arise naturally and logically in this theory. A transcendental equation determining the distribution function of anyons is obtained in terms of the statistics parameter, whose limiting values 0 and 1 correspond to Bosons and Fermions respectively. The distribution function is determined as a power series involving the Boltzmann factor and the statistics parameter and we also express the distribution function as an infinite continued fraction. The last form enables one to develop approximate forms for the distribution function, with the first approximant agreeing with our earlier investigation.
\end{abstract}

Electronic address: ${ }^{a}$ Raghunath.acharya@asu.edu, ${ }^{b}$ pswamy@siue.edu

August 2003

PACS 05.30.-d, 05.90.+m, 05.30.Pr 


\section{INTRODUCTION}

We formulate a theory of particles obeying intermediate statistics, interpolating between Bosons and Fermions, which might be called anyons. Our formulation will be based on two assumptions: 1) The exchange symmetry or permutation of the coordinates of the particles in the many particle wave function is accompanied by multiplication by a complex number $f$, thus generalizing the symmetric or antisymmetric wave functions and 2) The principle of Detailed Balance: if $n_{1}, n_{2}$ represent the average occupation numbers of states labelled by 1 and 2 , then the number of transitions flowing from 1 to 2 must equal that flowing from 2 to 1 at equilibrium.

The particles described by this theory may or may not be the same as the anyons obeying intermediate or fractional statistics discussed in the literature. The objects named anyons [1-8] carry both an electric charge and a magnetic flux. They have attracted a great deal of attention and have been the subject of intense investigation in the literature. The anyons arise from the special circumstance in $2+1$ space-time dimensions, where the permutation group is the braid group and the Chern-Simons theory provides a natural realization of the anyons $[9,10]$. There has been a great deal of discussion in the literature on the thermostatistics of anyons [11]. Since the real world is strictly in a $3+1$ dimensional space, anyons may not be real particles: they could be quasi-particles playing important roles in condensed matter phenomena. More recently, the subject of generalized statistics has been investigated in one dimension in the context of many kinds of statistics [12]. In contrast to the theory of anyons familiar in the literature, our present approach is not limited to two space dimensions and is valid in the real world of $3+1$ dimensional space-time. It is important to point out that this is due to the fact that we do not invoke the spin-statistics theorem and do not require ordinary spin to be interpreted in two dimensional space.

On one hand, the theory based only on Detailed Balance does not have the features of the full-fledged theory of anyons which takes advantage of the braid group in two dimensions. On the other hand, in this formulation we investigate the idea of interpolating statistics only in the context of the statistical mechanics of a gas in equilibrium, without the constraints imposed by quantum field theory. Here we shall use the name anyons, for convenience, to refer to particles obeying intermediate statistics, with the understanding that the present work needs to be developed further by incorporating additional assumptions before the connection with true anyons described in the literature could be established.

The subject of anyons has been well investigated in the literature, especially in the context of quantum field theory and the braid group $[2,5,7,8]$. Interesting results have also been derived to describe the thermostatistics of anyons, such as determining the virial coefficients $[11,13]$. However, the theoretical basis of the statistical mechanics of anyons has not really been established. For instance, the distribution function for the anyons has not been determined in an exact form. We had used an ansatz for the distribution function in our earlier work in order to derive many of the thermodynamic properties [11]. More recently, Chaturvedi and Srinivasan [14] have done a comparative study of the different interpolations between Bose-Einstein (BE) and Fermi-Dirac (FD) statistics available in the 
literature, in the context of the most general interpolations, including Haldane statistics and Gentile statistics, with the conclusion that the distribution function introduced by us [11] has some desirable features.

Consequently it is worthwhile to investigate the basic theoretical structure of anyons from the point of view of statistical mechanics and investigate the distribution function of the anyons if possible. This is the goal of this paper. Not only shall we determine the distribution function of the particles obeying the interpolation statistics, we shall also formulate a theory which leads to this determination without resorting to the restriction to a two dimensional space. Our formulation will be based on the quantum theory of many particles permitting a generalized interpolating exchange symmetry statistics with no other assumptions. Remarkably, this formulation leads to an exact theory which requires the employment of basic numbers.

In Sec.II we study the scattering probability of many particle states obeying interpolating statistics. We establish the fact that the basic numbers arise naturally and logically in this theory. We introduce the method of Detailed Balance in Sec. III and derive a transcendental equation for the distribution function in terms of the Boltzmann factor and the statistics determining parameter. The distribution function of anyons is studied in Sec. IV where we show that a closed form solution is not possible. We present the exact solution for the distribution function as an infinite series as well as in the form of an infinite continued fraction which is amenable to approximations. Sec. V contains a brief summary and conclusions.

Let us introduce an ensemble of particles, the anyons, which obey a generalized statistics, interpolating between (BE) and (FD) statistics. We begin with the framework for building the wave functions of these anyons by a generalized procedure of $f$-symmetrizing in such a way that it will reduce, in appropriate limits, to the standard procedure of symmetrizing for Bosons and anti-symmetrizing for Fermions. The operation of permutation or exchange of the coordinates of the many particle wave function results in multiplication by the complex number $f$, the exchange symmetry parameter, so that

$$
\mathcal{P} \Psi_{n}\left(\cdots, q_{i}, \cdots, q_{j}, \cdots\right)=f \Psi_{n}\left(\cdots, q_{j}, \cdots, q_{i}, \cdots\right) .
$$

Since the Hamiltonian has the property $\mathcal{P}^{-1} \mathcal{H} \mathcal{P}=\mathcal{H}$, it follows that $\mathcal{H}\left(\mathcal{P} \Psi_{n}\right)=E_{n}\left(\mathcal{P} \Psi_{n}\right)$ and thus $\mathcal{P} \Psi_{n}$ is an eigenfunction of the Hamiltonian with the same eigenvalue as $\Psi_{n}$. Thus $f \Psi_{n}$ is proportional to $\Psi_{n}$ and consequently we may take $|f|^{2} \Psi_{n}=\Psi_{n}$. Hence the general exchange symmetry that would lead to an intermediate statistics could be implemented by the complex number $f$ with the property $|f|^{2}=1$. We shall accordingly choose the exchange symmetry to be implemented by $f=e^{i \pi \alpha}$, where $\alpha$ is the statistics determining parameter, $0 \leq \alpha \leq 1$, so that $f^{*}=f^{-1}$. The limits $f \rightarrow 1,-1$ correspond respectively to the $\mathrm{BE}$ and FD statistics representing Bosons and Fermions. This procedure for incorporating exchange symmetry among anyons is justified [7] by the special property of rotations in two dimensions. Here we may treat it as an assumption or an ansatz, not restricted to two space dimensions but valid in any number of dimensions. 


\section{MANY PARTICLES AND QUANTUM PROBABILITIES}

Following Feynman [15], we consider the two particle scattering amplitude defined by the product $a_{1} b_{2}$, where $a_{1}=\langle 1 \mid a\rangle$ describes the scattering of particle $a$ into state 1 and $b_{2}=\langle 2 \mid b\rangle$ describes the process $b \rightarrow 2$. We shall take 1 and 2 to be the same state at the end in order to deal with identical particles. The exchange symmetry has to do with the process corresponding to $a \rightarrow 2, b \rightarrow 1$ which is indistinguishable from the direct process and the amplitude for this process would be $f a_{2} b_{1}$ due to the exchange factor $f$. The total probability amplitude is the sum of the direct and exchange processes. Employing the abbreviation $\langle 1 \mid a\rangle=\langle 2 \mid a\rangle=a$, we find the probability of this two particle scattering process involving non-identical particles to be

$$
p_{\text {non }}^{(2)}=\left(1+|f|^{2}\right)|a|^{2}|b|^{2}=2\left(|a|^{2}|b|^{2}\right),
$$

since $|f|^{2}=1$. This probability is the same as for ordinary Bosons [15]. However, for identical particles, we need to take account of interference between the two processes and that makes a great deal of difference. We obtain the probability in this case to be

$$
p_{\text {identical }}^{(2)}=\left(1+|f|^{2}+f+f^{-1}\right)|a|^{2}|b|^{2},
$$

since $f^{*}=f^{-1}$ and this probability depends on the statistics determining parameter $\alpha$. In the limit $f \rightarrow 1$, this would reduce to the case of Bosons and it would be twice as much as in Eq.(2) for the non-identical particles. For arbitrary $f$ the probability for the process involving identical particles relative to that for non-identical particles is given by

$$
P_{\text {identical }}^{(2)}=\frac{1}{2}\left(2+f+f^{-1}\right)|a|^{2}|b|^{2} .
$$

In what follows, we shall omit $|a|^{2},|b|^{2}$ etc. since the single particle states are properly normalized. Understanding this to be a proportionality, we might henceforward refer to this itself as the probability for the process, or just the probability of the two particle state, for simplicity.

Similarly, we consider the three-particle processes $a \rightarrow 1, b \rightarrow 2, c \rightarrow 3$ together with the exchange processes with factor $f$ for each exchange operation, thus resulting in the combination $a b c+f a c b+f b a c+f^{2} b c a+f^{2} c a b+f^{3} c b a$ with the same abbreviation as earlier. We can now determine the probability for the three particle process, or just for the three particle state with identical particles, as

$$
P^{(3)}=\frac{1}{6}\left(1+2 f^{-1}+2 f^{-2}+f^{-3}\right)\left(1+2 f+2 f^{2}+f^{3}\right) .
$$

We can reduce this to the form

$$
\begin{aligned}
P^{(3)} & =\frac{1}{6}\left\{10+8\left(f+f^{-1}\right)+4\left(f^{2}+f^{-2}\right)+\left(f^{3}+f^{-3}\right)\right\} \\
& =\frac{1}{6}\left\{6+7\left(f+f^{-1}\right)+4\left(f^{2}+f^{-2}+1\right)+\left(f^{3}+f^{-3}+f+f^{-1}\right)\right\} .
\end{aligned}
$$


Recognizing the pattern here, we observe that the right hand side in the expressions for the probabilities in Eqs.(4), (5), (6) contain basic numbers [16], with the base $f$. They are indeed expressed succinctly in terms of the basic numbers defined by

$$
[n]_{f}=\frac{f^{n}-f^{-n}}{f-f^{-1}} .
$$

We shall henceforward omit the subscript $f$ for simplicity. Here $f=e^{i \pi \alpha}$, the BE limit is $\alpha \rightarrow 0, f \rightarrow 1$ and the FD limit is $\alpha \rightarrow 1, f \rightarrow-1$ and $f^{*}=f^{-1}$. Our formulation is symmetric under $f \rightarrow f^{-1}$ and is the familiar generalization of the basic numbers introduced long ago by F. H. Jackson [16]. Studying the limits we find that the Bose limit gives $[n] \rightarrow n$ while the Fermi limit is quite different: $[n] \rightarrow(-1)^{n+1} n$ which becomes $-n$ for even numbers and $+n$ for odd numbers. In this limit, we are therefore dealing with a generalization of ordinary fermions for which the exclusion principle is not automatically valid: in the FD limit it can be imposed by hand only. Returning to the basic numbers introduced in Eq.(7), it is quite evident in Eqs.(4), (5), (6), and as will be seen in more detail below, that the basic numbers arise naturally, automatically, in the theory of particles obeying interpolating statistics. This feature is quite new and not recognized in the standard theory of anyons in the literature. This might be an indication that the theory of interpolating statistics naturally involves a deformation of the system such as that described by the basic number system with its consequences, without however introducing a deformed algebra of operators.

In addition to the representation,

$$
[n]=f^{n-1}+f^{n-3}+\cdots+f^{-n+3}+f^{-n+1},
$$

the following representation of the bracket number

$$
[n]=\frac{\sin n \pi \alpha}{\sin \pi \alpha},
$$

will be found most useful. In terms of the basic numbers, the probabilities may now be expressed conveniently and succinctly as

$$
\begin{aligned}
& P^{(2)}=\frac{1}{2}(2+[2]), \\
& P^{(3)}=\frac{1}{6}(6+7[2]+4[3]+[4]), \\
& P^{(4)}=\frac{1}{4 !}(35+54[2]+52[3]+36[4]+18[5]+6[6]+[7]),
\end{aligned}
$$

and so on. We can derive a useful result,

$$
[1]+[3]+[5]+\cdots+[2 n-1]=([n])^{2} .
$$

which can be proved by using [17] the identity,

$$
\frac{1}{\sin t} \sum_{k=1}^{n} \sin (2 k-1) t=\left(\frac{\sin n t}{\sin t}\right)^{2} \text {. }
$$


We shall also find the following result quite useful:

$$
[n-1][n]=[2]+[4]+[6]+\cdots[2(n-1)] .
$$

In other words, $[2][3]=[2]+[4],[3][4]=[2]+[4]+[6]$, etc. This is proved by using the identity

$$
\sum_{k=0}^{n-1} \sin k y=\sin \frac{(n-1) y}{2} \sin \frac{n y}{2} \csc \frac{y}{2} .
$$

These identities can be used to re-express Eqs.(10) in the form

$$
\begin{aligned}
& P^{(2)}=\frac{1}{2}(2+[2]), \\
& P^{(3)}=\frac{1}{3 !}(2+[2])(2+2[2]+[3]), \\
& P^{(4)}=\frac{1}{4 !}(2+[2])(2+2[2]+[3])(2+2[2]+2[3]+[4]) .
\end{aligned}
$$

In this manner, generalizing to $n$ particles, we are led to the probability for the $\mathrm{n}$-anyon state:

$$
P^{(n)}=\frac{1}{n !}(2+[2])(2+2[2]+[3]) \cdots(2+2[2]+2[3]+\cdots+2[n-1]+[n]) .
$$

\section{DETAILED BALANCE}

From Eq.(16), we can infer the enhancement factor, which is a measure of how much greater the probability of the $n+1$-particle state is, compared to the probability of the $n$-particle state. For now, we simply express it as

$$
F(n)=\frac{P^{(n+1)}}{P^{(n)}},
$$

which can be determined from Eq.(16). This enhancement factor provides the essential step in the method of Detailed Balance. This brings us to the second assumption upon which our theory of interpolating statistics rests: if $n_{1}, n_{2}$ represent the average occupation numbers of states 1 and 2 respectively, then the number of transitions flowing from 1 to 2 must equal that flowing from 2 to 1 at equilibrium. This is the principle of Detailed Balance. We should stress that this principle is characteristic of thermodynamic equilibrium and may be regarded as a consequence of the second law of thermodynamics [18]. Indeed the principle of Detailed Balance is valid when thermodynamic equilibrium prevails or the validity of microscopic reversibility in the language of statistical physics. This notion is based on the reversibility of the microscopic equations of motion, or on the Hermitian nature of the scattering Hamiltonian [19]. The principle of Detailed Balance can thus be stated as

$$
n_{1} F\left(n_{2}\right) e^{\beta E_{1}}=n_{2} F\left(n_{1}\right) e^{\beta E_{2}},
$$


where the population of each level is governed by the Boltzmann factor and $F(n)$ is the enhancement factor. This yields

$$
\frac{n}{F(n)} e^{\beta E}=\text { constant }=z
$$

which would, in principle, enable us to determine the distribution function for the anyons in terms of the Boltzmann factor. In the BE limit for instance, the enhancement factor is just $n+1$, which immediately leads to the BE distribution

$$
n=\frac{1}{z^{-1} e^{\beta E}-1} \text {. }
$$

where $z=e^{\beta \mu}$ is the fugacity of the gas. Let us now proceed in the same manner for arbitrary $f$. The enhancement factor for arbitrary $f$ is given by Eqs.(16) and (17) which reduces to the simple form

$$
F(n)=\frac{P^{(n+1)}}{P^{(n)}}=\frac{1}{n+1}(2+2[2]+2[3]+\cdots 2[n]+[n+1]) .
$$

To proceed further, we employ the result

$$
\sum_{k=0}^{n}[k]=2 \cos (\pi \alpha / 2)[n / 2][(n+1) / 2],
$$

which is easy to prove by using identities involving sums of trigonometric functions. This gives us the following result for $F(n)$, after some algebra:

$$
F(n)=\frac{1}{n+1} 2 \cos (\pi \alpha / 2)[(n+1) / 2]\{[n / 2]+[n / 2+1]\} .
$$

We can also derive another identity

$$
[n / 2]+[n / 2+1]=2 \cos (\pi \alpha / 2)[(n+1) / 2]
$$

which can be used to simplify the above result. Hence we obtain

$$
F(n)=\frac{4}{n+1}\{[(n+1) / 2] \cos (\pi \alpha / 2)\}^{2} .
$$

We observe that this reproduces the expected result $F(n) \rightarrow n+1$ in the Bose limit. Upon now invoking the Detailed Balance we obtain the important result

$$
\frac{1}{z} e^{\beta E}=F(n) / n=\frac{4}{n(n+1)}\{[(n+1) / 2] \cos (\pi \alpha / 2)\}^{2} .
$$

This can be rewritten in the following convenient form in order to deal with the distribution function:

$$
e^{\beta(E-\mu)}=\frac{1}{n(n+1)} \frac{\sin ^{2}(n+1) \pi \alpha / 2}{\sin ^{2} \pi \alpha / 2} .
$$


Solving this equation should, in principle, lead to the distribution function for the anyons in this formulation. At this point before dealing with the distribution function, we need to study the nature of the intermediate statistics as an interpolation between the BE and FD limits. Specifically we need to study the limits corresponding to BE and FD statistics.

We have already observed that $F(n) \rightarrow n+1$ in the Bose limit, $f \rightarrow 1$. Indeed, it is readily verified that Eq.(26) reproduces the correct BE statistical distribution. The case of Fermi limit, $\alpha \rightarrow 1, f \rightarrow-1$, is, however, somewhat complicated. We have: $[n] \rightarrow n,-n$ for odd and even occupation numbers respectively. Evaluating the limit in Eq.(26), the enhancement factor may be put in the form,

$$
\lim _{\alpha \rightarrow 1} F(n)=\frac{1}{n+1}\left(\mathcal{I} m e^{i(n+1) \pi / 2}\right)^{2},
$$

thus resulting in a generalized Fermion theory. This corresponds to an infinite dimensional representation analogous to the generalized fermions investigated by Chaichian et al . If we consider only $n=0,1$, and impose the exclusion principle "by hand", then $F(n) \rightarrow 1,0$. This is equivalent to $F(n) \rightarrow 1-n$ and in this case it reproduces the standard Fermi distribution with the exclusion principle. For arbitrary $n$, which is allowed, however, it is an infinite value representation

$$
\lim _{\alpha \rightarrow 1} F(n)=\frac{1}{n+1}\{1,0,1,0,1, \cdots\}
$$

and we find that $F(n)$ has repeating values $1 /(n+1)$ and 0 in the limit $\alpha \rightarrow 1, f \rightarrow-1$. This is thus a special and interesting feature of this theory. Thus for arbitrary values of the parameter $f$, including the Fermi limit $f \rightarrow-1$, the theory of intermediate statistics requires the existence of generalized fermions beyond the exclusion principle.

\section{THE DISTRIBUTION FUNCTION}

We begin by rewriting Eq.(27) in the form

$$
e^{\beta(E-\mu)}=\frac{1}{n(n+1)} \frac{\sin ^{2}(n+1) x}{\sin ^{2} x} .
$$

Here $x=\pi \alpha / 2$ in terms of the statistics determining parameter. The object is to determine the average occupation number $n$, the distribution function, so that we can compare with the standard BE or FD distribution and understand the nature of the interpolating statistics. We can expand the right hand side in a power series

$$
e^{\beta(E-\mu)}=\frac{1}{n}+a_{0}+a_{1} n+a_{2} n^{2}+a_{3} n^{3}+\cdots
$$

where 


$$
\begin{aligned}
& a_{0}=\csc ^{2} x\left(x \sin 2 x-\sin ^{2} x\right) \\
& a_{1}=\csc ^{2} x\left(x^{2} \cos 2 x-x \sin 2 x+\sin ^{2} x\right) \\
& a_{2}=\csc ^{2} x\left\{-x^{2} \cos 2 x-\sin ^{2} x+\left(x-2 x^{3} / 3\right) \sin 2 x\right\} \\
& a_{3}=\csc ^{2} x\left\{\left(x^{2}-x^{4} / 3\right) \cos 2 x+\sin ^{2} x-\left(x-2 x^{3} / 3\right) \sin 2 x\right\},
\end{aligned}
$$

and so on. The coefficients to any desired order can be obtained by using Mathematica. It is clear that $a_{0} \rightarrow 1$, while $a_{n} \rightarrow 0$ for $n \geq 1$ in the Bose limit, which is consistent with the BE distribution as described in Sec. III. In the case of the Fermi limit, $a_{0} \rightarrow-1$ and it leads to the generalized fermions. We can rewrite the above as

$$
\frac{1}{g}=n-\frac{a_{1}}{g} n^{2}-\frac{a_{2}}{g} n^{3}-\frac{a_{3}}{g} n^{4}-\cdots .
$$

where $g=e^{\beta(E-\mu)}-a_{0}$. This series can be reverted to express $n$ as a series in powers of $1 / g$, thus

$$
n=1 / g+B / g^{2}+C / g^{3}+D / g^{4}+E / g^{5}+F / g^{6} \cdots
$$

where

$$
\begin{aligned}
& B=a_{1} / g, \quad C=\left(2 a_{1}^{2} / g^{2}+a_{2} / g\right), D=\left(5 a_{1} a_{2} / g^{2}+a_{3} / g+5 a_{1}^{3} / g^{3}\right), \\
& E=E=6 a_{1} a_{3} / g^{2}+3 a_{2}^{2} / g^{2}+14 a_{1}^{4} / g^{4}+a_{4} / g+21 a_{1}^{2} a_{2} / g^{3}, \\
& F=7 a_{1} a_{4} / g^{2}+7 a_{2} a_{3} / g^{2}+84 a_{1}^{3} a_{2} / g^{4}+a_{5} / g+28 a_{1}^{2} a_{3} / g^{3}+28 a_{1} a_{2}^{2} / g^{3}+42 a_{1}^{5} / g^{5},
\end{aligned}
$$

and so on. Upon rearrangement, we can rewrite the above form for the distribution function as the following series:

$$
\begin{aligned}
n(E) & =1 / g+a_{1} / g^{3}+a_{2} / g^{4}+\left(2 a_{1}^{2}+a_{3}\right) / g^{5} \\
& +\left(5 a_{1} a_{2}+a_{4}\right) / g^{6}+\left(5 a_{1}^{3}+6 a_{1} a_{3}+3 a_{2}^{2}+a_{5}\right) / g^{7}+\cdots .
\end{aligned}
$$

We observe that this is a power series in $1 / g$ but the term with $1 / g^{2}$ is absent. We can rewrite Eq.(36) in the form

$$
n(E)=1 / g+\sum_{k=3}^{\infty} \alpha_{k} / g^{k}
$$

where

$$
\alpha_{3}=a_{1}, \quad \alpha_{4}=a_{2}, \quad 2 a_{1}^{2}+a_{3}=\alpha_{5}, \quad 5 a_{1} a_{2}+a_{4}=\alpha_{6},
$$

etc. We can now express this in the form of a continued fraction. We invoke the standard algorithm which can be introduced as follows [21]. If a continued fraction is of the form

$$
\frac{B_{n}}{C_{n}}=c_{0}+\frac{b_{1}}{c_{1}+\frac{b_{2}}{c_{2}+\cdots}},
$$


then the successive convergents (approximants) are obtained by the recurrence formulae

$$
B_{n}=c_{n} B_{n-1}+b_{n} B_{n-2}, \quad C_{n}=c_{n} C_{n-1}+b_{n} C_{n-2}, \quad B_{-1}=1, C_{-1}=0 .
$$

Thus in order to put the series in Eq.(37) in the continued fraction form, we proceed with the first convergent and set $c_{0}=B_{0} / C_{0}=0$. Using the recurrence relations, Eq.(40), we determine $B_{0}=0, C_{0}=1, b_{1}=1, c_{1}=g, B_{1}=1, C_{1}=g$. This determines the first convergent as $n=1 / g$. Next, the recurrence relations lead to $b_{2}=-\alpha_{3} g, c_{2}=g^{2}+\alpha_{3}, B_{2}=$ $g^{2}+\alpha_{3}, C_{2}=g^{3}$ which determines the second convergent to be

$$
n=\frac{1}{g-\frac{\alpha_{3} g}{g^{2}+\alpha_{3}}} .
$$

Continuing on with successive convergents in this manner, we obtain the desired infinite continued fraction form for the distribution function as follows

$$
n(E)=\frac{1}{g-\frac{\alpha_{3} g}{g^{2}+\alpha_{3}-\frac{\alpha_{4} g}{\alpha_{3} g+\alpha_{4}-\frac{\alpha_{5} g}{\alpha_{4} g+\alpha_{5}-\cdots}}}}
$$

This is the distribution function in the exact theory, albeit not in a closed form. Approximations can be implemented in order to investigate the thermostatistical properties of the anyons. The first approximant,

$$
n(E)=\frac{1}{e^{\beta(E-\mu)}-a(\alpha)},
$$

was indeed employed, as an ansatz, in our earlier investigation [11]. It might be pointed out that the continued fraction form is mathematically desirable in view of the fact that it can be expressed as a ratio of polynomials, hence analytic in the variable $\alpha$ as well as offering the best approximation possible. This form is also amenable to investigation in terms of Pade approximants.

Finally it must be pointed out that Eq.(42) is an exact expression for the distribution function, albeit in the form of an infinite continued fraction, and can be determined explicitly for any specific value of the parameter $\alpha$, either as an infinite continued fraction, or to any desired approximation. For instance, for the case of $\alpha=\frac{1}{2}$, the exact form of the distribution function is

$$
\left.n(E)\right|_{\alpha=\frac{1}{2}}=\frac{1}{g+\frac{a_{0} g}{g^{2}-a_{0}+\frac{\left(a_{0}-\pi^{3} / 48\right) g}{a_{0} g-\left(a_{0}-\pi^{3} / 48\right)+\cdots}}},
$$

where $a_{0}\left(\frac{1}{2}\right)=\pi / 2-1$. 


\section{SUMMARY}

The thermodynamic distribution function of anyons in two space dimensions, obeying interpolating statistics is not known in the literature and is an open question. We have not only found an answer, thus determining the distribution function, we have also demonstrated that the theory describing interpolating statistics has several remarkable and interesting features. Our investigation leads to a generalized definition of permutation symmetry in arbitrary dimensions and not restricted to two space dimensions. We have shown that the theory of permutation symmetry that would describe particles obeying interpolating statistics is succinctly formulated in the language of basic numbers. These basic numbers arise naturally and automatically in this formulation but do not explicitly invoke any deformed oscillator algebra. Our theory is based on the principle of Detailed Balancing which is a consequence of the Second law of thermodynamics. Furthermore, this formulation leads to the determination of the exact distribution function, without having to introduce any approximation. In this manner, we have formulated the theory which leads to a transcendental equation for the distribution function of anyons in terms of the statistics determining parameter and the Boltzman factor containing the energy of the state. We obtain a solution of this equation which we express as a power series as well as in the form of a continued fraction. We show that the first approximation of this theory reproduces a form of the distribution function introduced by us in an earlier investigation.

An important feature of our formulation consists of the fact that the basic numbers arise naturally and automatically in this theory, specifically the symmetric formulation of the basic numbers. The basic numbers arise automatically in our theory but they are not part of an oscillator algebra, nor do we introduce the construction of Fock space for the particles obeying the intermediate statistics. The theory reduces to BE statistics in the Bose limit, $f \rightarrow 1, \alpha \rightarrow 0$. The Fermi limit, $f \rightarrow-1, \alpha \rightarrow 1$ leads to generalized fermions beyond the exclusion principle and thus correspond to infinite dimensional representation and they reduce to the familiar FD statistics only when $n$ is restricted to 0,1 by hand.

Although the algebra of q-oscillators appears in the literature on the subject of anyons $[7,8,20]$, it is also known that q-oscillators may have nothing to do with anyons since the former exist in arbitrary space-time dimensions. In our formulation, we do not use q-oscillator algebra, neither do we use Fock states. We do not make use of the Hamiltonian other than to recognize that the Hamiltonian should be of a form that permits Detailed Balance. The Hamiltonian consists of only the kinetic energy terms and is the free Hamiltonian [5] in the anyon gauge and the anyon wave functions satisfy "twisted" boundary conditions.

It is interesting that the first approximant (first convergent) of our solution corresponds to the approximate form for the distribution function

$$
n(E)=\frac{1}{e^{\beta E-\mu)}-a(\alpha)}
$$

which is the form introduced in an earlier investigation [11]. It is interesting that this also agrees with the partition function derived by the method of Green function [20], to first order 
approximation. It should be stressed that while the form above is a point of agreement for an approximate theory, the form of $a$ in the present formulation as in Eq.(32), as a function of the statistics determining parameter, is very different from that of [11]. Finally we obtain the exact form of the distribution function for the case $\alpha=\frac{1}{2}$ in the form of a continued fraction which can be evaluated to any desired order. Such a form for the distribution function can indeed be obtained for any chosen value of $\alpha$, the statistics determining parameter

It is of much interest that this formulation leads to an intermediate statistical mechanics, interpolating between $\mathrm{BE}$ and $\mathrm{FD}$, without explicit use of the special properties valid in $2+1$ space-time dimensions, but only stems from the principle of Detailed Balance. This contrasts with investigations in the literature of anyons which arise from the braid group in the special case of $2+1$ space-time. As is seen from Eq.(30), the dependence on the statistics determining parameter is through a quadratic function, hence invariant under $\alpha \rightarrow-\alpha$, implying a clockwise-counterclockwise symmetry of the braid [6]. It would be worthwhile to continue this investigation further in order to explore the relation to these anyons. As this formulation reveals several desirable and interesting theoretical features, it is appropriate to pose some questions such as the following. Does the generalization to any space dimensions imply that the connection with Chern-Simons type of gauge theory can be extended beyond two space dimensions? Is it possible to formulate the partition problem corresponding to this generalized, exact theory? Is it possible to determine the many useful thermodynamic properties of the system described by such an exact theory? These questions will occupy us in further investigations of this theory. 


\section{REFERENCES}

[1] J. Leinnas and J. Myrheim, Nuovo Cimento B 37, (1977) 1.

[2] F. Wilczek (ed.)Fractional Statistics and Anyon Superconductivity, World Scientific (1991), Singapore.

[3] S. Forte, Rev. Mod. Phys. 64 (1992) 193.

[4] S.S. Chern et al,(ed.) Physics and Mathematics of Anyons, World Scientific (1991), Singapore.

[5] A. Lerda, Anyons, Springer (1992), Berlin.

[6] A. Khare,Fractional Statistics and Quantum Theory, World Scientific Pub. (1998) Singapore.

[7] M. Frau et al, arXiv:hep-th/9407161 v1, July 1994.

[8] L. Frappat et al, Phys. Lett. B 369 (1996) 313.

[9] F.Wilczek, Phys.Rev.Lett,48,(1982) 114; Phys.Rev.Lett,49,(1982) 957.

[10] R. Jackiw and S. Templeton, Phys.Rev.,D23, (1981) 2291; C.R. Hagen, Ann. Phys., 157, (1984) 342.

[11] R. Acharya and P. Narayana Swamy, J. Phys. A; math. Gen.A 27, (1994) 7247; See also other references therein.

[12] A. Polychronakos, Les Houches Lectures, Summer 1998, <arXiv;hep-th/9902157>, February 1999.

[13] D. Arovas et al, Nuc. Phys. B 251 (1985), 117; See ref.( [11]) for a full list of references.

[14] S. Chaturvedi and V. Srinivasan, Physica A 246 (1997), 576.

[15] R.P. Feynman, R. B. Leighton and M. Sands, The Feynman Lectures on Physics, Addison-Wesley Pub. Co.(1965), Reading MA.

[16] H. Exton, q-Hypergeometric functions and applications, Ellis Horwood Ltd.(1983) Chichester.

[17] I.S.Gradshteyn and I.M.Ryzhik, Table of Integrals, Series and products, Academic press (1980) New York.

[18] S-K Ma, Statistical Mechanics, World Scientific Publishing (1985) Singapore; L.E. Reichl, A Modern Course in Statistical Physics, second edition, John Wiley \& sons Inc.(1998), New York; P.T. Landsberg, editor, Problems in thermodynamics and statistical physics, Pion Limited (1971), London.

[19] D.C. Mattis, Statistical Mechanics Made Simple, World Scientific Publishing, (2003) Singapore.

[20] M. Chaichian, R.G. Felipe and C. Montonen, J. Phys. A: Math.Gen.A 26 (1993) 40174034.

[21] G. E. Andrews, R. Askey and R. Roy, Special Functions, Cambridge University Press, (2001) New York. 